\title{
Effect of force control algorithms on the scanning probe microscope lithography system
}

\author{
Jia-Yush Yen ${ }^{\text {a) }}$ and I-Ming Lin \\ Department of Mechanical Engineering, National Taiwan University, Taipei, 10617 Taiwan, \\ Republic of China \\ Chih-Kung Lee \\ Institute of Applied Mechanics, National Taiwan University, Taipei, 10617 Taiwan, Republic of China
}

(Received 15 October 2004; accepted 14 December 2004; published online 1 March 2005)

\begin{abstract}
This letter addresses the servo issue in the scanning probe microscope (SPM) lithography. The performance of the probe controller affects the ability of the lithography system to form uniform marks on the sample surface. Different control algorithms are tested on a commercial SPM system. The tests include the controller from the original system, a proportional integral differential controller, and a modern $\mu$ controller. The design procedure for the $\mu$ controller is described. The experimental results show that carefully tuned controllers are able to suppress the undesirable vibrations, and the $\mu$ controller achieves the most uniform lithography marks. (C) 2005 American Institute of Physics. [DOI: 10.1063/1.1863932]
\end{abstract}

The scanning probe microscope (SPM) relies on a very sharp probe to trace the sample surface at an atomic scale. In the case of SPM lithography, the controller maintains a constant probe force to scratch the sample surface. Because the SPM probe can be made as small as $10 \mathrm{~nm}$ in diameter, the SPM lithography can achieve lithography marks with nanometer accuracy. This feature has made it a very attractive tool for many applications. In the case of integrated circuit lithography, the SPM probe is used either to discharge current onto the photoresist ${ }^{1,2}$ or to mechanically plow through the photoresist ${ }^{3,4}$ to leave a nano precision circuitry pattern. In both cases, high performance probe controllers would be essential. The design of high performance controllers usually depends on good system models. However, it would be very difficult in this case because of the interactive forces described by the relative geometry of the tip and the sample surfaces. ${ }^{5-7}$ A robust control algorithm that can suppress the model uncertainties and counter the disturbance forces would then be necessary. Most of the previous results have treated the atomic force microscope (AFM) probe control for topography. This letter investigates the effect of the control on the SPM lithography. The test specimen is a hard polycarbonide surface. The commercial controller, a proportional integral differential (PID) controller and a modern $\mu$ controller are experimentally tested, and the experimental results show that poor servo design often results in unpredictable lithography marks. It may even introduce serious chattering in the process. A properly tuned $\mu$ controller is found to be most suitable to treat the case. The synthesis procedure is also described for reference.

The test system is based on an AutoProbe LS AFM system from Park Scientific Instruments. The system consists of a standard cantilever probe and laser light lever design. The piezoelectric scanner drives the necessary $x, y$, and $z$ move-

\footnotetext{
${ }^{\text {a) }}$ Author to whom correspondence should be addressed; electronic mail: jyen@ntu.edu.tw
}

ment. The probe in this experiment is $3 \mu \mathrm{m}$ thick, $28 \mu \mathrm{m}$ wide and $225 \mu \mathrm{m}$ long. The nominal force constant is $2.8 \mathrm{~N} / \mathrm{m}$ and the resonant frequency is $75 \mathrm{kHz}$. Typical tip radius is about $10 \mathrm{~nm}$. To measure a good reference model, the system identification procedure is carried out with an Agilent 23670A dynamic signal analyzer. The input to the system is the piezoelectric tube voltage and the output is the PSPD signal, both measured in volts. The measured frequency response shows the first resonance occurs at about $600 \mathrm{~Hz}$. This is a result of the probe suspension. The signal coherence indicates that the measurement is no longer accurate above the frequency of $800-900 \mathrm{~Hz}$.

The purpose of this study is to investigate the servo effects on the SPM lithography. However, tuning the PID controller is well known and this letter will only describe the $\mu$ synthesis procedure. Consider a practical system, $\widetilde{G}$, belonging to the set $M\left(G, W_{u}\right)=\left\{\max _{\omega} G\left(I+\Delta G W_{u}\right):|\Delta G(j \omega)| \leqslant 1\right\}$, where $G$ is the nominal system, $W_{u}$ is a multiplicative weighting function, and $\Delta$ is a transfer function satisfying $\max _{\omega}|\Delta(j \omega)| \leqslant 1$. The $\mu$ synthesis procedure computes for a

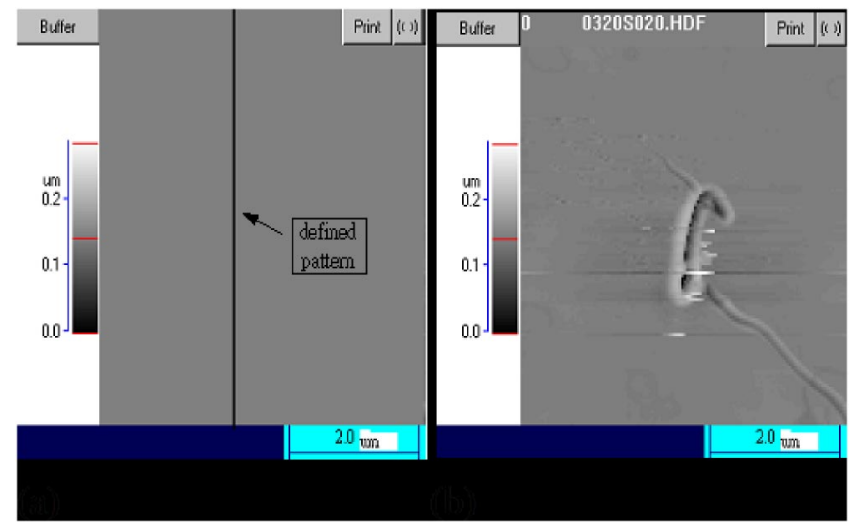

FIG. 1. (Color online) (a) Desired lithography pattern, (b) actual lithography result with commercial controller. 
robust controller $K$ that achieves the criterion $\| W_{p}(I$ $+G K)^{-1} \|_{\mu}<1$ for all $\widetilde{G} \in M\left(G, W_{u}\right)$. The weighting function $W_{p}$ in the inequality can then serve as a performance specification. Therefore, the uncertainty weighting is set to $W_{u}(s)=(s+691) /(s+18850)$ and the performance weighting are designed to be $W_{p}(s)=(2.5 s+785) /(s+31416)$ to ensure good performance as high as $1 \mathrm{KHz}$. The $\mu$ design tool in MATLAB then results in a sixth order controller

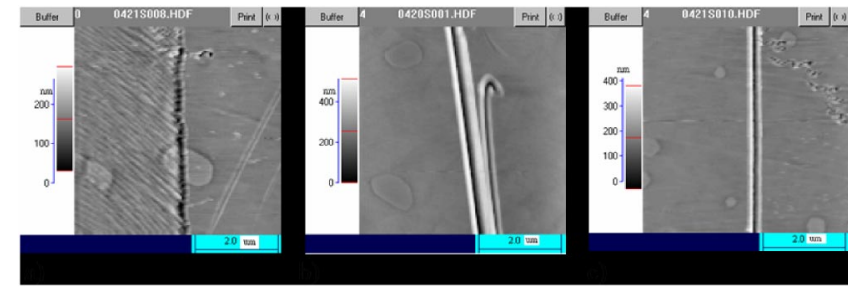

FIG. 3. (Color online) Lithography results using PID controller.

$$
K(s)=\frac{17.7 s^{2}+3.98 \times 10^{5} s^{4}+2.0 \times 10^{9} s^{3}+1.9 \times 10^{13} s^{2}+3.1 \times 10^{16} s+1.7 \times 10^{20}}{s^{6}+1.3 \times 10^{4} s^{5}+1.1 \times 10^{8} s^{4}+4.1 \times 10^{11} s^{3}+1.8 \times 10^{15} s^{2}+2.3 \times 10^{18} s+9.0 \times 10^{16}}
$$

Figure 1 shows the nanolithography software interface from Park Scientific Instruments (PSI). The interface enables one to load a topographic picture from the ProScan Data and define the lithography patterns [Fig. 1(a)]. Figure 1(b) shows the resulted lithographic pattern which does not at all resemble the desired pattern. The hook shape at the beginning of lithography mark is caused by the familiar cantilever bend when it was extended too fast. ${ }^{8}$ Obviously, there is plenty of room for effective servo improvement.

Figure 2 shows the relation between the applied forces and the resulting depths of the grooves. The applied forces are recorded from the position-sensitive photodetector (PSPD) control signal set points. The depths are obtained by averaging the depths of the center of the grooves. The dotted curve shows the result for the PSI controller. There is hardly any relationship to be deduced except that larger forces probably do result in deeper cuts. The data from the PID and the $\mu$ controller (dashed and solid curves), on the other hand, show a clear relationship to enable the control over the lithography depths. The force-depth relationship can be described by $y=0.056 x+2.29$ in the case of the PID control, and $y=0.055 x+1.82$ in the case of the $\mu$ controller. It is also

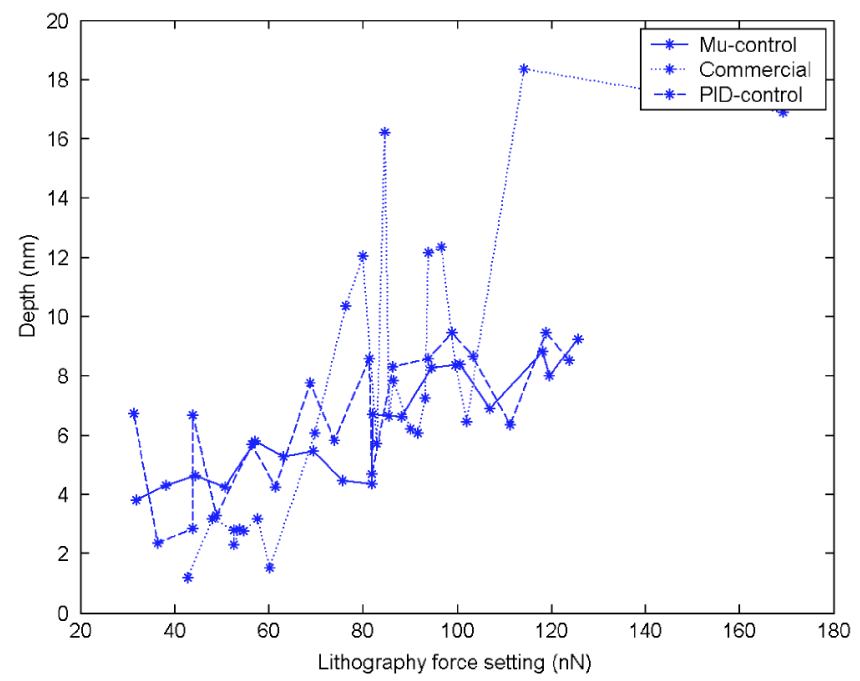

FIG. 2. Force-depth relationship for (a) the PSI controller, (b) the PID controller, (c) the $\mu$ controller. observed that the $\mu$ controller achieves less variation from the fitted curve. Figure 3 shows the topography of the resulting grooves for the PID controller. Serious chattering [Fig. 3(a)] and the hook-like scratch mark [Fig. 3(b)] can both occur if the PID controller is not properly tuned. A fairly good control can be obtained [Fig. 3(c)] with proper tuning. The result from the $\mu$ controller (Fig. 4) provides a very uniform lithography pattern.

For a closer comparison, the topography along the grooves is shown in Fig. 5. The thick solid line represents the bottom topography of the $\mu$-control groove. The average cut depth is $5.3 \mathrm{~nm}$ and the standard deviation is $1.1 \mathrm{~nm}$. The dashed line represents the bottom topography from the PID controller. The average cut depth is $6.8 \mathrm{~nm}$ and the standard deviation in this case increases to $2.5 \mathrm{~nm}$. It is obvious that the $\mu$ controller offers more uniform groove topography. This is a result that is not very obvious by just inspecting the two-dimensional contour plot or the three-dimensional topography. It is believed that a controller that directly ad-

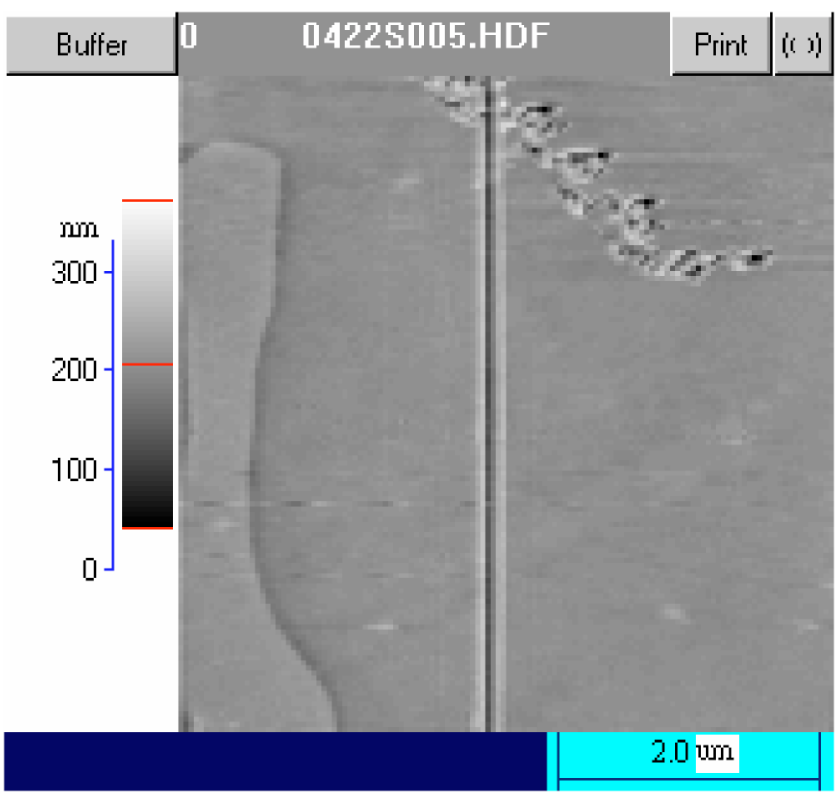

FIG. 4. (Color online) Lithography result using $\mu$ controller. 


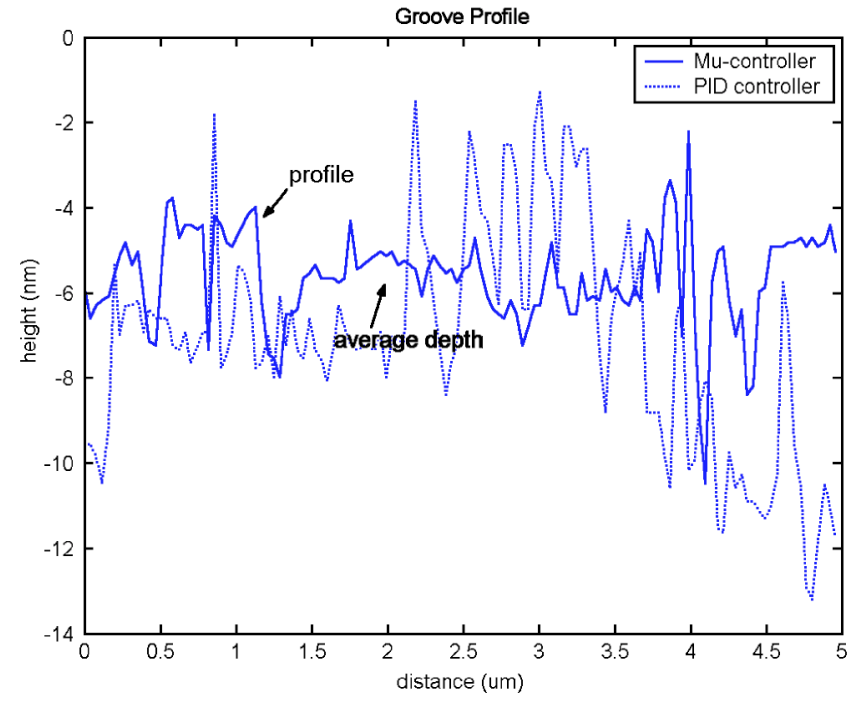

FIG. 5. The groove profile from the $\mu$ controller and the PID controller. dresses the disturbance force can more adequately compensate for the force variation induced from the sample/tip interaction.

This research is supported in part by the National Science Council, Taiwan, under Contract No. NSC92-2213-E002-042, and in part by the Ministry of Economy, Taiwan, under Contract No. 91-EC-17-A-05-S1-0017.

${ }^{1}$ S. H. Y. Yamamoto and H. Tokumoto, Jpn. J. Appl. Phys., Part 1 34, 3396 (1995).

${ }^{2}$ L. L. Sohn and R. L. Willett, Appl. Phys. Lett. 67, 1552 (1995).

${ }^{3}$ O. M. El Rifai and K. Youcef-Toumi, Proceedings of the 2002 IEEE International Conference on Control Applications, Glasgow, Scotland, UK, 18-20 September 2002, p. 1126.

${ }^{4}$ B. Klehn and U. Kunze, J. Appl. Phys. 85, 3897 (1999)

${ }^{5}$ L. G. Hector and S. R. Schmid, Wear 215, 247 (1998).

${ }^{6}$ O. M. El Rifai and K. Youcef-Toumi, Proceedings of the IEEE/ASME International Conference on Advanced Intelligent Mechatronics, Como, Italy, 8-12 July 2001, p. 1325.

${ }^{7}$ K. Maekawa and A. Itoh, Wear 188, 115 (1995).

${ }^{8}$ O. M. El Rifai and K. Youcef-Toumi, Proceedings of the American Control Conference, Chicago, Illinois, 28-30 June 2000, p. 2118. 